\title{
AVALIAÇÃO DA ETAPA DE DESCASQUE DE TORRETES DE EUCALIPTO PARA POLPA
}

\section{EVALUATION OF DEBARKING ON EUCALIPTO WOOD FOR PULP}

\author{
Maurem Kayna Lima Alves ${ }^{1} \quad$ Odilon Oliveira Ferreira $^{2}$
}

\begin{abstract}
RESUMO
Este trabalho teve por objetivo analisar a etapa de descasque de madeira para polpa em uma empresa de celulose e papel do Rio Grande do Sul, abrangendo os itens: (1) caracterização do aproveitamento do tempo; (2) quantificação das capacidades de produção teórica e efetiva; e (3) determinação dos custos de produção. A metodologia baseou-se no estudo de tempos operacionais e capacidades de produção. A maior causa de interrupções são os problemas mecânicos, seguidos das pausas técnicas e as esperas e demoras, que juntas chegam a $87,46 \%$ do total de interrupções nesta operação, eqüivalendo a $17 \%$ do tempo de trabalho efetivo para uma eficiência operacional de $81 \%$, que pode ser considerada satisfatória, pois está próxima ao possível de ser obtido para o nível de mecanização. Os custos com mão-de-obra operacional representaram a maior parcela dos custos diretos da operação.
\end{abstract}

Palavras-chave: Eficiência operacional, descasque mecanizado, eucalipto.

\begin{abstract}
This paper aims to analyze the debarking of wood for pulp in a pulp and paper manufacturingcompany in Rio Grande do Sul State. The analysis comprised the following items: (1) characterization of time's utilization; (2) quantifying of the theoretical and effective production capacities; and (3) determination of production costs. The methodology was based in the study of the operational times and capacities of production. The greatest cause of interruptions are the mechanical problems, followed by technical pauses and expectations and delays, which mean, together, $87,46 \%$ of the total interruptions in this operation, that is, $17 \%$ of the effective time of working for an operational effectiveness of $81 \%$. These are satisfactory because it is almost the possible to be obtained for the mechanization level. The costs in personal to operate the system represented the greatest item of the direct costs of operation.
\end{abstract}

Key words: Operational efficiency, mechanized debarking, eucalyptus.

1. Eng. Florestal, MSc. em Engenharia Agrícola/CCR/UFSM. Rua Venâncio Aires, 2288/303. CEP: 97010-004. Santa Maria. RS.

2. Eng. Florestal, MSc., Prof. Titular do Departamento de Engenharia Rural/CCR/UFSM. CEP: 97105-900. Santa Maria. RS. 


\section{INTRODUÇÃO}

As fases de colheita e transporte de madeira são, segundo VILLELA FILHO (1992), importantes elos entre a floresta vista como matéria-prima (fibra in natura) e o mercado consumidor da fibra processada, seja na forma de celulose, papel, toras, chapas ou qualquer outra forma de beneficiamento da madeira.

Um sistema de abastecimento industrial de madeira, como também podem ser chamados os sistemas de colheita de madeira, de acordo com SANT'ANNA JÚNIOR (1992), consta de três atividades ou operações básicas: corte / derrubada; baldeio / arraste e transporte. Estas podem conter sub-operações, como desgalhamento, seccionamento e outros e contam com atividades de apoio, como o planejamento, controles operacionais e de custos e recursos humanos.

A avaliação dos sistemas de colheita de madeira, independente do grau de mecanização utilizado, é uma ferramenta fundamental para correções ou qualquer alteração do processo de produção, visando a racionalização e otimização dos recursos utilizados. Trata-se ainda de instrumento indispensável na comparação de diferentes métodos ou equipamentos.

Há dois parâmetros básicos que são interdependentes para a avaliação da eficiência de um sistema de produção: produtividade e custos. Entretanto, estes não são os únicos fatores a serem considerados, principalmente em se tratando de atividades dependentes de recursos naturais renováveis. Os aspectos relacionados à conservação do ambiente e às condições de trabalho do homem são de suma importância.

VIVAS (1996) apresentou a situação de uma empresa na Argentina que modificou seu sistema de produção e, portanto, necessitava avaliar esse novo sistema utilizando os parâmetros produtividade e custos. $\mathrm{O}$ método adotado para determinar a eficiência baseava-se no preenchimento de planilhas com informações sobre a utilização do tempo e a produção de cada máquina.

Cabe ressaltar que, enquanto não ocorre a substituição dos sistemas manuais ou semimecanizados por sistemas totalmente mecanizados, não devem ser desprezadas as avaliações e o controle operacional como instrumento para otimização do sistema vigente. Os testes operacionais e a busca do desenvolvimento e aperfeiçoamento de equipamentos e sistemas adaptados à situação de cada empresa são o caminho para atingir níveis crescentes de produtividade, menores custos e qualidade do processo e do produto.

O empenho em otimizar o rendimento de sistemas de produção pouco mecanizados deve ser constante, pois deve-se considerar que há determinadas condições topográficas e/ou edáficas que praticamente impedem a mecanização com os equipamentos atualmente disponíveis. Assim, uma certa parcela da produção provavelmente ainda continuará a ser executada com métodos similares ou derivados dos atualmente empregados por um período de tempo relativamente longo.

SEIXAS (1986) em artigo sobre planejamento e estudo de sistemas de exploração, ressalta a necessidade de se conhecer profundamente as atividades desenvolvidas em uma operação florestal, sendo que o levantamento de informações deve servir de base para qualquer interferência que se queira fazer. BRIDI \& FERLIN (1994) também ressaltam a importância dos testes operacionais e 
das adaptações para as situações específicas de cada empresa como instrumento para a melhoria contínua.

MALINOVSKI (1992) argumenta que a mecanização das atividades florestais nem sempre é a melhor prática para se racionalizar o trabalho, pois é necessário que haja combinação de fatores técnicos, econômicos e ambientais. Em muitos casos, uma modificação no decurso do trabalho nas atividades de colheita de madeira pode trazer melhores resultados que a implantação de processos mecanizados.

Segundo MACHADO (1989) e MARCON (1989) o índice mínimo de eficiência operacional do maquinário envolvido na colheita de madeira deve ser $70 \%$. Uma das formas de manter elevado o índice de disponibilidade mecânica, que influencia diretamente o aproveitamento do tempo disponível para o trabalho, é um programa de manutenção preventiva eficiente. MARCON comenta que a adoção de planos para a substituição de frotas e reformas, bem como a racionalização do uso dos equipamentos assegura maior vida útil e segurança operacional.

A eficiência operacional, para Gibson et al. citado por SANTOS et al. (1995), depende da disponibilidade mecânica e da utilização da máquina. Sendo que a disponibilidade é o tempo em que a máquina está apta a trabalhar e a utilização da máquina é o tempo em que a mesma está efetivamente produzindo.

ANDERSSON \& LAESTADIUS (1987) afirmam que a eficiência de um sistema de colheita de madeira é altamente dependente do ambiente em que é operacionalizado. Os principais fatores a serem considerados são: o clima, o terreno, a espécie vegetal, a infra-estrutura, o nível de desenvolvimento, a tradição do sistema utilizado e a estrutura da indústria. Os autores também citam algumas formas de expressar a eficiência da atividade de colheita no que se refere ao maquinário, dentre elas estão: o consumo de potência diário para produzir e transportar um determinado volume de madeira, ou ainda a relação do consumo de combustível para uma determinada potência demandada. Pode-se considerar também a produtividade do trabalho, expressa em homens $/ \mathrm{dia} / \mathrm{m}^{3}$ produzido, quando o sistema envolve menores níveis de mecanização; ou ainda a eficiência econômica em termos de custo por unidade produzida (metros cúbicos, metros esteres, toneladas).

Uma das alternativas para reduzir os custos de produção é incrementar a capacidade de produção. Isso é especialmente relevante nas atividades de colheita florestal, uma vez que representam um dos custos mais elevados da empresa (SILVA \& MACHADO, 1995).

Gibson et al. citado por SANTOS et al. (1995 a), afirmam que o custo da colheita florestal mecanizada pode aumentar substancialmente caso a manutenção das máquinas e equipamentos seja deficiente ou inadequada. Isso ocorre porque a qualidade da manutenção afeta diretamente a eficiência operacional do equipamento e, consequentemente, o custo de produção.

$\mathrm{Na}$ Escandinávia há um preceito que vem sendo adotado desde que os salários dos trabalhadores florestais se tornaram relativamente altos: "No man on the ground" ou seja, nenhum homem no chão (WÄSTERLUND, 1994).

No Brasil, embora a mão-de-obra não qualificada ainda seja bastante mal remunerada, os custos com este fator de produção têm sido incrementados pela evolução dos custos sociais. 
MOREIRA (1992) cita um aumento de 308\% (de 35 a 108\% do salário pago ao trabalhador) nos custos sociais no período de 1970 a 1992. Segundo ele, este índice pode ser maior ou menor de acordo com a política de cada empresa, porém este aumento, aliado a bolsões de falta de mão-deobra, foi determinante para viabilizar a mecanização das operações florestais, em função do maior rendimento operacional oferecido por esta.

Ainda com relação ao fator mão-de-obra, um ponto importante a considerar é o treinamento. Para o sucesso da colheita florestal há que se combinar máquinas com boa eficiência, serviços de assistência técnica local e operadores e mecânicos bem preparados (LUNDQVIST, 1996). Investir em treinamento significa reduzir custos de produção, riscos de acidente e danos ao ambiente.

MACHADO et al. (1990) classificam os custos em diretos, indiretos e casuais. Os custos diretos são os relacionados com a mão-de-obra, materiais e outras despesas que afetam diretamente o custo total. Indiretos seriam os custos tais como aluguel, seguros, impostos e depreciação. Já os custos casuais são aqueles esporádicos, tais como multas ou prêmios por atraso ou entrega antecipada, respectivamente. Porém, há outros métodos de cálculo que consideram os custos com seguro e depreciação como custos diretos (MACHADO, 1994).

O custo operacional dos equipamentos é a base de cálculo para as avaliações econômicas e estudos comparativos entre sistemas, através da variação das grandezas de seus parâmetros. Os seus componentes são: valor de aquisição; vida útil; valor residual; taxa de remuneração; seguros e outras taxas; utilização anual; mão-de-obra; combustível ou energia e manutenção (RODRIGUEZ et al., 1992).

O objetivo deste trabalho foi avaliar o descasque de toretes de eucalipto para polpa realizado em prestadoras de serviço de uma empresa de celulose e papel do Estado do Rio Grande do Sul, sem quaisquer intervenções no processo produtivo, aplicando e adaptando métodos de avaliação e conceitos encontrados na literatura.

A análise abrangeu os seguinte parâmetros:

(1) a caracterização do aproveitamento do tempo;

(2) a quantificação das capacidades de produção teórica e efetiva; e

(3) a determinação dos custos diretos de produção.

\section{MATERIAIS E MÉTODOS}

\section{Descrição do objeto de estudo}

Os povoamentos onde foram feitos os levantamentos eram formados por Eucalyptus saligna com idade entre 7,5 e 8,5 anos. No geral o relevo era levemente ondulado a ondulado. A avaliação estendeu-se de agosto a novembro de 1996.

$\mathrm{Na}$ empresa onde se realizou o presente estudo, a produção de madeira é totalmente terceirizada e subdividida, havendo uma ou mais prestadoras de serviço para cada etapa da 
produção. Neste trabalho, serão apresentados os resultados referentes à etapa de descasque dos toretes.

\section{Coleta de dados}

Foram acompanhadas as atividades de duas prestadoras de serviço: a empresa A, no município de Barra do Ribeiro - Horto Florestal Barba Negra e a empresa B, no município de Butiá Hortos Leão I e Corrêa da Silva. Essas empresas pertencem aos mesmos proprietários.

Os dados relativos ao aproveitamento de tempo dos conjuntos mecanizados de descasque foram coletados em planilhas específicas mediante o acompanhamento diário das operações com a cronometragem de tempos operacionais, anotando todas as interrupções ao trabalho, com respectivas causas.

Quanto à produção, o levantamento foi realizado de duas formas: (1) estabeleceu-se um tempo de acompanhamento que cobrisse todas as etapas de operação, inclusive os deslocamentos inerentes à mesma, assim, em baterias de cinco minutos não consecutivos (332 unidades amostrais) foram contados os toretes descascados e (2) foram feitos acompanhamentos da operação com registro do tempo transcorrido, através de cronometragem, onde, após a obtenção de duas a três pilhas de madeira descascada, todos os toretes descascados no período foram cubados pelo método de Huber (FINGER, 1992).

As informações necessárias para efetuar a estimativa dos custos de produção foram obtidas com a aplicação de questionários em cada empresa. Utilizou-se, ainda, estimativas do consumo de combustível por conjunto trator-descascador; dados das planilhas de aproveitamento de tempo e preços levantados em revendas de equipamentos.

\section{Características técnicas da operação}

São mantidas sempre uma ou duas máquinas reserva para substituição de alguma que necessite de manutenção corretiva. Os descascadores são da marca Valmet e os tratores utilizados são Valmet 980 e Massey Ferguson MF 292, na Barra do Ribeiro e em Butiá, respectivamente. As gruas utilizadas em ambos os casos são da marca Madal.

\section{Determinações}

\section{Aproveitamento de tempo}

Foi elaborado um quadro contendo um resumo das informações obtidas no campo, no qual figuram os tempos de observação, jornadas total, teórica e efetiva, tempo total de interrupções e a classificação de acordo com a natureza da interrupção, em valores absolutos e percentuais do total. A partir desses dados, foram calculados os índices de aproveitamento de tempo.

$\mathrm{R}_{1}$ - representa o aproveitamento do tempo da jornada total de trabalho em relação à jornada teórica; expressa, basicamente, o tempo gasto com a viagem do local de saída na cidade até o horto. 


$$
\mathrm{R}_{1}(\%)=\frac{\text { Jte }}{\text { Jtot }} \cdot 100 \quad \text { ou: } \quad \mathrm{R}_{1}(\%)=\frac{\text { Tte }}{\text { Hobs }} \cdot 100
$$

$\mathrm{R}_{2}$ - expressa a relação entre a jornada efetiva e a total, representando, além do tempo gasto na viagem até o horto, o tempo consumido nos deslocamentos acampamento - talhão, as paradas por chuvas e todas as interrupções ocorridas ao longo da jornada.

$$
\mathrm{R}_{2}(\%)=\frac{\text { Jef }}{\text { Jtot }} \cdot 100 \quad \text { ou: } \quad \mathrm{R}_{2}(\%)=\quad \frac{\text { Tef }}{\text { Hobs }} \cdot 100
$$

$R_{3}$ - mostra a relação entre a jornada efetiva e a teórica, expressando o aproveitamento do tempo realmente disponível para o trabalho (eficiência operacional). Este é o mais significativo índice de desempenho operacional e pela análise das suas causas, se desenvolve o processo de otimização de uma determinada atividade ou sistema de trabalho.

$$
\mathrm{R}_{3}(\%)=\frac{\text { Jef }}{\text { Jte }} \cdot 100 \quad \text { ou: } \quad \mathrm{R}_{3}(\%)=\frac{\text { Tef }}{\text { Tte }} \cdot 100
$$

\section{Capacidade de produção}

A capacidade de produção teórica (CPT) refere-se ao volume produzido, em número de árvores, número de toretes, metros esteres ou metros cúbicos por hora, considerando-se um período de tempo efetivamente trabalhado, como se a eficiência operacional fosse de $100 \%$, isto é, sem considerar as interrupções. A capacidade de produção efetiva (CPE) é obtida pela multiplicação do modelo ajustado ou dos valores estimados de capacidade de produção teórica pelo fator de eficiência operacional $\left(\mathrm{R}_{3}\right)$.

Neste caso determinou-se a produção por unidade de tempo de duas formas e comparou-se os resultados através do teste " $t$ " de Student. A produção estimada através da contagem de toretes descascados por unidade de tempo foi convertida a metros esteres pelo fator de conversão adotado pela empresa e corrigida em função da eficiência operacional verificada.

Também estimou-se a produção por análise de regressão dos dados obtidos diretamente da cubagem dos toretes descascados num determinado período de tempo, onde a variável dependente era o número de toretes descascados e a variável independente o tempo consumido.

\section{Custos de produção}

Estimou-se os custos de produção para cada prestadora de serviço, com base no método adotado por FERREIRA (1980). Primeiramente, obteve-se o custo operacional por hora de cada equipamento, composto por custos fixos e variáveis. O custo de produção foi obtido dividindo-se o custo operacional do equipamento $(\mathrm{R} \$ / \mathrm{h})$ pela capacidade de produção verificada $(\mathrm{st} / \mathrm{h})$, chegandose ao valor consumido por unidade de produção $(\mathrm{R} \$ / \mathrm{st})$. Convém ressaltar que os valores obtidos são estimativas que referem-se apenas aos custos diretos. 
Todos os componentes dos custos fixos foram calculados em reais por hora de trabalho, considerando-se a jornada total. Para o cálculo dos custos variáveis, o consumo de combustíveis e lubrificantes corresponde às horas efetivas de trabalho e foi informado pelas próprias empresas.

\section{Análises estatísticas}

As análises estatísticas foram feitas, basicamente, através da avaliação e/ou comparação de médias, desvios padrão e coeficientes de variação, sendo que a comparação de médias foi feita depois de comprovada a normalidade dos dados pelo Teste de Lilliefors. Utilizou-se, ainda, análise de regressão e foram determinados intervalos de confiança para os valores referentes à capacidade de produção. Os softwares utilizados nos cálculos e análises foram o Excel 5.0 e Plot-It 3.0.

\section{RESULTADOS E DISCUSSÃO}

\section{Interrupções e aproveitamento do tempo}

A caracterização das interrupções na operação dos conjuntos de descasque é ilustrada pelo gráfico da Figura 1. Vale ressaltar que algumas das médias dessas interrupções apresentaram desvio padrão muito elevado. Nestes casos, sua ocorrência não pode ser atribuída a nenhuma variável independente do sistema de trabalho, mas sim ao acaso. O tempo total de interrupções representou, em média, $10,64 \%$ da jornada total de trabalho, $13,56 \%$ da jornada teórica e 17,39\% da jornada efetiva. São valores relativamente baixos, o que se confirma nos coeficientes de aproveitamento de tempo. No entanto, os coeficientes de variação, muito altos de acordo com PIMENTEL GOMES (1990), indicam a grande variabilidade desses valores.

As jornadas de trabalho teórica e efetiva foram de $8 \mathrm{~h}$ e $6 \mathrm{~min}$ e $6 \mathrm{~h}$ e $30 \mathrm{~min}$, respectivamente, sendo que o coeficiente de variação dos dados pode ser classificado como pequeno, pois fica abaixo dos $10 \%$ em ambos os casos, 4,33 e 9,55, respectivamente.

O aproveitamento médio da jornada total em termos de jornada teórica foi $77,82 \%$; de jornada efetiva foi $62,37 \%$. Já o aproveitamento da jornada teórica para trabalho efetivo chegou a $81,00 \%$. Nota-se que a eficiência operacional apresentou valor elevado, se comparada com a literatura: 56\% nos trabalhos de SANTOS \& MACHADO (1995) e SANTOS et al. (1995).

\section{Capacidade de produção}

Os valores referentes ao número de toretes descascados por unidade de tempo têm os resultados apresentados na Tabela 1. A estimativa de produção feita com base no número de toretes descascados por unidade de tempo (dados obtidos em 332 unidades amostrais) e o equivalente em metros esteres resultou numa produção média de 31,05 st/hora de trabalho, sem interrupções, que ajustada pelo fator de eficiência operacional $(81,00 \%)$ resulta numa produção efetiva média de 25,15 st/hora. O intervalo de confiança para os dados, com uma probabilidade de erro de $5 \%$ é de 24,50 a 
25,80 st/hora/equipe. Considerando a jornada teórica média observada $(8,09$ h) a produção efetiva média por dia no período avaliado foi, aproximadamente, 204 st/equipe.

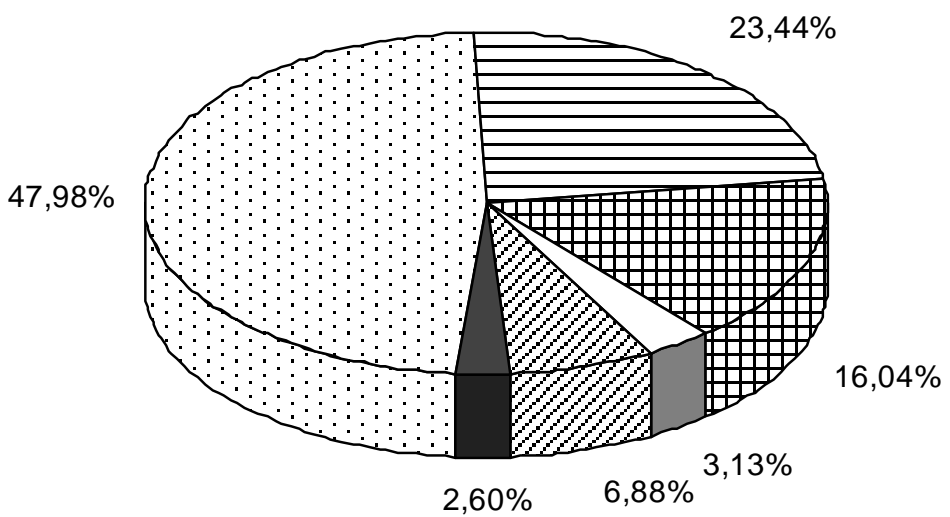

$\begin{array}{ll}\square \text { Pmec - falhas mecânicas } & \square \text { Ireg - pausas técnicas } \\ \square \text { Ides - interrupções por esperas e demoras } & \square \text { Idiv - interrupções por causas diversas } \\ \square \text { lpes - interrupções de ordem pessoal } \quad \square \text { lop - falhas operacionais }\end{array}$

FIGURA 1: Distribuição das interrupções por diferentes causas na atividade de descascamento.

Os dados de produção determinados através da cubagem, pelo método de Huber, dos toretes descascados permitiram calcular, por análise de regressão, a equação 4, para estimar o volume, em função do tempo de trabalho de descascamento. A equação apresentou coeficiente de determinação $\left(\mathrm{R}^{2}\right)$ de 0,699 .

$\mathrm{CPE}\left(\mathrm{m}^{3} / \mathrm{h}\right)=[0,1476+20,61 \times$ tempo $(\mathrm{h})] \times 0,81$

A produção efetiva média verificada por este método foi $16,8417 \mathrm{~m}^{3} /$ hora/equipe para a situação de eficiência operacional observada $(81 \%)$. Utilizando-se os fatores de conversão para metros esteres, dos povoamentos envolvidos, tem-se a produção efetiva média de 24,85 st/hora/equipe e 201,04 st/dia. O intervalo de confiança dos dados, com probabilidade de 5\%, é de 24,77 a 24,93 st/hora/equipe.

A comparação da estimativa de capacidade de produção obtida pelos diferentes métodos, mostrou , de acordo com o teste " $t$ " de Student, que não há diferença significativa entre os dois valores estimados. Entretanto, como o coeficiente de determinação da equação que forneceu os valores no segundo método de estimativa da produção foi baixo $(0,699)$, consideraremos para efeito de cálculo dos custos de produção a estimativa foi obtida pelo primeiro procedimento.

Como a eficiência operacional verificada nesta etapa da produção é elevada, seria difícil incrementar a capacidade de produção com estes mesmos equipamentos. Melhorias sempre são possíveis, mas como neste caso as interrupções são pequenas, o incremento resultante da redução destas seria pouco expressivo. 
TABELA 1: Número de toretes descascados nos períodos de amostragem e equivalências de produção por hora.

\begin{tabular}{l|c|c|c|c|c|c}
\hline \multicolumn{1}{c|}{ Talhão / Horto } & $\begin{array}{c}\text { Tempo } \\
(\mathrm{min})\end{array}$ & Toretes & $\begin{array}{c}\text { Toretes / } \\
\text { min }\end{array}$ & $\begin{array}{c}\text { Toretes / } \\
\text { hora }\end{array}$ & $\begin{array}{c}\text { st / hora } \\
\text { CPT }\end{array}$ & $\begin{array}{c}\text { st / hora } \\
\text { CPE }\end{array}$ \\
\hline 205 - Barba Negra & 4,91 & 62,40 & 12,65 & 759,15 & 28,87 & 23,34 \\
206 - Barba Negra & 4,89 & 62,60 & 13,05 & 782,83 & 32,75 & 26,50 \\
03- Leão I & 5,00 & 62,53 & 12,51 & 750,41 & 30,85 & 24,94 \\
04- Leão I & 5,00 & 64,56 & 12,91 & 774,75 & 32,17 & 25,99 \\
05- Leão I & 5,03 & 62,92 & 12,53 & 751,68 & 30,56 & 24,71 \\
06- Leão I & 5,00 & 53,67 & 10,73 & 644,00 & 25,35 & 20,50 \\
\hline Média Geral & - & - & 12,60 & 753,77 & 31,05 & 25,10 \\
Desvio Padrão & - & - & 2,58 & 154,75 & 6,54 & 5,29 \\
C.V. & - & - & 20,48 & 20,53 & 21,06 & 21,08 \\
\hline
\end{tabular}

\section{Custos de produção}

\section{Empresa A}

Custos fixos $-\mathrm{C}_{\mathrm{F}}=\mathrm{R} \$ 0,802 / \mathrm{h}$

Custos variáveis $-\mathrm{C}_{\mathrm{V}}=\mathrm{R} \$ 26,32 / \mathrm{h}$

Custo operacional - $\mathrm{C}_{\mathrm{O}}=\mathrm{C}_{\mathrm{F}}+\mathrm{C}_{\mathrm{V}}=\mathrm{R} \$ 27,12 / \mathrm{h}$

Para a capacidade de produção observada no descasque, o custo por metro estere descascado e pronto para o baldeio é de $\mathrm{R} \$ 1,09$ / st.

$\mathrm{Na}$ empresa A, o maior percentual do custo operacional refere-se aos custos com mão-deobra operacional $(32,95 \%)$, seguidos do custo com peças de reposição, que representaram 30,97\% do custo operacional; a mão-de-obra gerencial e para manutenção respondeu por 13,35\%; o custo com combustível representou 11,5\%; o consumo de lubrificantes $4,06 \%$; o custo com pneus $3,36 \%$ e o custo com filtros $0,85 \%$. Os custos fixos representaram apenas $2,96 \%$ do total.

\section{Empresa B}

Custos fixos $-\mathrm{C}_{\mathrm{F}}=\mathrm{R} \$ 0,712$

Custos variáveis $-\mathrm{C}_{\mathrm{V}}=\mathrm{R} \$ 25,65 / \mathrm{h}$

Custo operacional - $\mathrm{C}_{\mathrm{O}}=\mathrm{C}_{\mathrm{F}}+\mathrm{C}_{\mathrm{V}}=\mathrm{R} \$ 26,36 / \mathrm{h}$

Para a capacidade de produção observada no descasque, os custos por metro estere descascado e pronto para o baldeio é de $\mathrm{R} \$ 1,10$ / st.

O comportamento dos componentes do custo operacional na empresa B não apresenta grandes diferenças em relação ao observado na empresa A. Há alguma diferença nos valores, mas a proporção de cada item sobre o total segue a mesma tendência, exceto o item mão-de-obra gerencial e para manutenção, que aqui é inferior ao custo com combustíveis, o que pode ser explicado pelo 
fato de não haver um supervisor de campo nesta empresa, pois tal função é desempenhada pelos proprietários. A distribuição dos percentuais é a seguinte: 36,84\% com mão-de-obra operacional; $31,87 \%$ com peças de reposição; $13,55 \%$ com combustivel; 7,93\% com mão-de-obra gerencial e para manutenção; $4,17 \%$ com lubrificantes; $2,39 \%$ com pneus; e $0,55 \%$ com filtros; enquanto os custos fixos representaram $2,7 \%$ do total dos custos operacionais.

Os valores de custo operacional de tratores + descascadores utilizados na empresa giram em torno de $\mathrm{R} \$ 45,00$. A discrepância em relação ao que é apresentado aqui refere-se à metodologia empregada no cálculo, ressaltando que os valores aqui apresentados referem-se apenas aos custos diretos.

\section{CONCLUSÕES}

A partir dos dados apresentados, analisados e discutidos, conclui-se que:

- as principais causas de interrupções à operação (problemas mecânicos e pausas técnicas), embora representem um percentual relativamente pequeno da jornada de trabalho, podem ser reduzidas;

- o índice de eficiência operacional é elevado; porém, com os equipamentos atualmente utilizados é difícil obter algum aumento deste índice ou da capacidade produtiva;

- os custos com mão-de-obra operacional representam a maior parcela do total dos custos diretos da operação.

\section{REFERÊNCIAS BIBLIOGRÁFICAS}

ANDERSSON, Stig; LAESTADIUS, Lars. Efficiency in highly mechanized wood harvesting systems. In: SIMPÓSIO SOBRE SISTEMAS EXPLORAÇÃO, TRANSPORTE, ERGONOMIA E SEGURANÇA EM REFLORESTAMENTOS. Anais... Curitiba : FUPEF, 1987. p. 133-143.

BRIDI, Gerson Luiz, FERLIN, Vianei. Melhorias técnicas e vantagens econômicas na atividade de colheita de madeira na Rigesa. VIII SEMINÁRIO DE ATUALIZAÇÃO SOBRE SISTEMAS DE COLHEITA DE MADEIRA E TRANSPORTE. FLORESTAL. Curitiba. Anais... Curitiba : FUPEF, 1994. p. 49-58.

FERREIRA, Odilon Oliveira. Anotações didáticas da disciplina de Mecanização Florestal (não publicado), Santa Maria : Universidade Federal de Santa Maria,1980.

FINGER, César Augusto Guimarães. Fundamentos de Biometria Florestal. 1. ed. Santa Maria: UFSM / CEPEF / FATEC, 1992. 269 p.

LUNDQVIST, Roland. Treinamento de operadores de máquinas florestais visando a redução de custo e proteção ambiental. In: IX SEMINÁRIO DE ATUALIZAÇÃO SOBRE SISTEMAS DE 
COLHEITA DE MADEIRA E TRANSPORTE FLORESTAL. Curitiba. Anais... Curitiba: UFPR / FUPEF, 1996. p. 127-132.

MACHADO, Carlos Cardoso. Exploração Florestal. VI Parte. Viçosa : Universidade Federal de Viçosa - Imprensa Universitária, 1989.

MACHADO, Carlos Cardoso. Planejamento e Controle de Custos na Exploração Florestal. Viçosa : Universidade Federal de Viçosa - Imprensa Universitária, 1994.

MACHADO, Carlos Cardoso; BARROS, Antônio A. A. de; VALE, Antônio Bartolomeu do. PERT-CPM: instrumento do planejados da exploração florestal. 1. ed. Viçosa : Universidade Federal de Viçosa - Imprensa Universitária, 1990.

MALINOVSKI, Jorge Roberto. O estágio atual da exploração florestal no Brasil. In: VII SEMINÁRIO DE ATUALIZAÇÃO SOBRE SISTEMAS DE EXPLORAÇÃO E TRANSPORTE FLORESTAL. Curitiba. Anais... Curitiba: UFPR / FUPEF, 1992. p.221-226.

MARCON, Eloi Jacob. A manutenção preventiva de equipamentos e veículos como fator de segurança na disponibilidade operacional. In: VI SEMINÁRIO DE ATUALIZAÇÃO SOBRE SISTEMAS DE EXPLORAÇÃO E TRANSPORTE FLORESTAL. Curitiba. Anais... Curitiba: UFPR / FUPEF, 1989. p. 217-224.

MOREIRA, Manuel Francisco. O desenvolvimento da mecanização na exploração florestal sob a ótica de custos. In: VII SEMINÁRIO DE ATUALIZAÇÃO SOBRE SISTEMAS DE EXPLORAÇÃO E TRANSPORTE FLORESTAL. Curitiba. Anais... Curitiba: UFPR / FUPEF, 1992. p.161-171.

PIMENTEL GOMES, Frederico. Curso de estatística experimental. 13. ed. Piracicaba : Nobel S.A., 1990. 468 p.

RODRIGUEZ, Augusto Valencia; CASTRO, Paulo Fernando de; DRUMOND, Geraldo Sinésio. Engenharia industrial aplicada à exploração florestal. In: VII SEMINÁRIO DE ATUALIZAÇÃO SOBRE SISTEMAS DE EXPLORAÇÃO E TRANSPORTE FLORESTAL. Curitiba. Anais... Curitiba: UFPR / FUPEF, 1992. p.40-66.

SANT'ANNA JÚNIOR, Mário. Tendências atuais e perspectivas futuras dos sistemas de extração florestal. In: VII SEMINÁRIO DE ATUALIZAÇÃO SOBRE SISTEMAS DE EXPLORAÇÃO E TRANSPORTE FlORESTAL. Curitiba. Anais... Curitiba: UFPR / FUPEF, 1992. p.137160.

SANTOS, Sérgio L. Martins, MACHADO, Carlos Cardoso, LEITE, Hélio Garcia. Análise técnicoeconômica da extração de eucalipto em áreas planas com o "forwarder". Revista Árvore. Viçosa, v. 19, n. 3, p. 213-227, 1995.

SEIXAS, Fernando. Planejamento e estudo de sistema de exploração florestal. IPEF. Piracicaba, v. 34, p25-30, 1986.

SILVA, Márcio Lopes da, MACHADO, Carlos Cardoso. Influência do diâmetro e do número de árvores por hectare no custo de corte do eucalipto. Revista Árvore. Viçosa, v. 19, n. 3, p. 308- 
318, 1995.

VILLELA FILHO, Adhemar. Economia florestal e interligações na exploração e transporte florestal. In: VII SEMINÁRIO DE ATUALIZAÇÃO SOBRE SISTEMAS DE EXPLORAÇÃO E TRANSPORTE FLORESTAL. Curitiba. Anais... Curitiba: UFPR / FUPEF, 1992. p.237265.

VIVAS, Pablo. N. La introducion y adaptacion del harvester en las plantaciones forestales de Papel Prensa. In: PRIMER SEMINARIO DE ACTUALIZACIÓN EN SISTEMAS DE COSECHA Y TRANSORTE FORESTAL. Eldorado, Misiones, Argentina. Actas... Eldorado: Universidad Nacional de Misiones/ Facultad de Ciencias Forestales, 1996. p. 27-36.

WÄSTERLUND, Iwan. Wood harvesting systems in Scandinavia and environmental aspects. VIII Seminário de Atualização sobre Sistemas de Colheita de Madeira e Transporte Florestal. Curitiba : FUPEF, 1994. 